\title{
Quantization of Chern-Simons theory à la loop quantum gravity
}

\section{G. Luchini ${ }^{* \dagger}$}

Instituto de Física de São Carlos - USP

E-mail: gabriel.luchini@usp.br

A. Rios

UFES

E-mail: riosfisica@gmail.com

C. P. Constantinidis

UFES

E-mail: cpconstantinidis@pq. cnpq.br

O. Piguet

UFES

E-mail: opiguetepq.cnpq.br

We consider the canonical loop quantization (following the loop quantum gravity (LQG) scheme) of Chern-Simons theory in a noncompact space with the topology of a cylinder.

At quantum level, constraints are defined and completely solved, and we obtain a gauge and diffeomorphism invariant Hilbert space, which is infinite dimensional but separable.

Some remarks about the inclusion of boundary are done.

4th International Conference on Fundamental Interactions -ICFI2010,

August 1-7, 2010

Viçosa, Brazil

\footnotetext{
* Speaker.

${ }^{\dagger}$ Financial support given by CNPq.
} 


\section{Introduction}

One approach to a quantum theory of gravitation is through the so called "loop quantization" [1]. Despite general relativity, it is reasonable to expect that such quantization method should also work for theories sharing basic features of this first one, such as diffeomorphism invariance and being fully constrained. Here we show how one can apply this quantization scheme to ChernSimons theory, regardless its relation to gravity. A detailed discussion and references are given at [3].

Notably we have here also the possibility to follow in great details the methods explained in books about the subject, which makes this work also interesting from the pedagogical point of view.

We shall mainly treat here the case where the 2-dimensional space manifold is a cylinder or, equivalently, the pierced real plane $\mathbb{R}^{2} \backslash\{0\}$. We shall also briefly consider the case of a disk with boundary.

\section{Canonical treatment of Chern-Simons theory}

The action reads [4]

$$
S=-\kappa \int_{\mathscr{M}} d^{3} x \varepsilon^{\mu \nu \rho} \operatorname{Tr}\left(A_{\mu} \partial_{v} A_{\rho}+\frac{2}{3} A_{\mu} A_{v} A_{\rho}\right)
$$

with $A=A_{\mu}^{I} \tau_{I} d x^{\mu}(\mu=0,1,2)$ being the dynamical field; a Lie algebra valued connection 1-form. The gauge group $G$ will be assumed to be compact and semi-simple.

General covariance is broken by the assumption that $\mathscr{M}=\Sigma \times \mathbb{R}$, i.e., by splitting spacetime into space $(\Sigma)$ and time, which leads us to

$$
S=-\kappa \int_{\mathbb{R}} \int_{\Sigma} d t d^{2} x \varepsilon^{a b} \operatorname{Tr}\left(\dot{A}_{a} A_{b}+A_{0} F_{a b}\right)
$$

where $F_{a b}^{I}=\partial_{a} A_{b}^{I}-\partial_{b} A_{a}^{I}+f_{J K}^{I} A_{a}^{J} A_{b}^{K}$ is the spatial restriction of the curvature $(a, b \ldots=1,2)$.

The Hamiltonian ${ }^{1}$ and the symplectic structure after Dirac-Bergman's algorithm then read

$$
H=G(\varepsilon) \equiv \int_{\Sigma} d^{2} x \varepsilon^{I}(x) G^{I}(x) \approx 0 \quad\left\{A_{a}^{I}(x), A_{b}^{J}(y)\right\}=\frac{1}{\kappa} \varepsilon_{a b} \delta^{I J} \delta^{2}(x-y)
$$

where $G^{I} \equiv-\frac{\kappa}{2} \varepsilon^{a b} F_{a b}^{I} \approx 0$ is the Gauss constraint, whose algebra (gauge group algebra) is

$$
\left\{G(\varepsilon), G\left(\varepsilon^{\prime}\right)\right\}=G\left(\varepsilon \times \varepsilon^{\prime}\right) \quad\left(\varepsilon \times \varepsilon^{\prime}\right)^{\prime} \equiv f_{J K}^{I} \varepsilon^{J} \mathcal{E}^{\prime K}
$$

We see that the system is fully constrained, and the spatial components $A_{1}^{I}$ and $A_{2}^{I}$ form a pair of conjugate variables. Diffeomorphism invariance follows from gauge invariance. Thus, implementation of Gauss constraint guarantees - in the classical regime - invariance under spatial diffeomorphism, that can be seen ${ }^{2}$ after calculating the Lie derivative of the canonical variable along a spatial vector $\xi: \mathscr{L}_{\xi} A_{a}=\xi^{b} F_{b a}+D_{a}\left(\xi^{b} A_{b}\right) \approx D_{a}\left(\xi^{b} A_{b}\right)$.

\footnotetext{
${ }^{1}$ It is the smeared Gauss constraint. The $\varepsilon(x)$ is a test function.

${ }^{2}$ This is equal to a gauge transformation with parameter $\xi^{a} A_{a}^{I}$.
} 


\section{The Hilbert space}

In order to get the quantum theory we need to construct the kinematical Hilbert space $\mathscr{H}_{\text {kin }}$ and then select the physical states by implementing the constraints.

We choose a specific topology for the space, as $\Sigma \sim \mathbb{R} \times S^{1}$, so the range of the coordinates are $z \in \mathbb{R}$ and $\theta \in[0,2 \pi]$. Then, canonical variables are defined as operators in the Schroedinger picture, satisfying

$$
\left[\hat{A}_{\theta}^{I}(x), \hat{A}_{z}^{J}(y)\right]=\frac{i}{\kappa} \delta^{I J} \delta^{2}(x-y)
$$

and the states are described by the wave functionals $\Psi\left[A_{\theta}\right]=\left\langle A_{\theta} \mid \Psi\right\rangle$. Field operators act on these states as

$$
\left\langle A_{\theta}\left|\hat{A}_{\theta}^{I}(x)\right| \Psi\right\rangle=A_{\theta}^{I}(x) \Psi\left[A_{\theta}\right] \quad\left\langle A_{\theta}\left|\hat{A}_{z}^{I}(x)\right| \Psi\right\rangle=\frac{1}{i \kappa} \frac{\delta}{\delta A_{\theta}^{I}(x)} \Psi\left[A_{\theta}\right] .
$$

Using this representation, a particular solution of the Gauss constraint $\hat{G}^{I} \Psi\left[A_{\theta}\right]=0$ is given by [2] $\Psi_{\circ}\left[A_{\theta}\right]=e^{2 \pi i \alpha_{\circ}}$ with

$$
\alpha_{\circ}=\frac{\kappa}{6 \pi} \int_{\tilde{\Sigma}} d^{3} x\left(\varepsilon^{\mu v \rho} \operatorname{Tr}\left(g^{-1} \partial_{\mu} g g^{-1} \partial_{\nu} g g^{-1} \partial_{\rho} g\right)\right)-\frac{\kappa}{2 \pi} \int_{\Sigma=\partial \tilde{\Sigma}} d^{2} x \operatorname{Tr}\left(A_{\theta} g^{-1} \partial_{z} g\right)
$$

where $g \in G$ is defined in terms of $A_{\theta}$ by $A_{\theta}=g^{-1} \partial_{\theta} g$. A general solution [2] is

$$
\Psi\left[A_{\theta}\right]=\Psi_{\circ}\left[A_{\theta}\right] \psi^{\operatorname{inv}}\left[A_{\theta}\right]
$$

where the functional $\psi^{\operatorname{inv}}\left[A_{\theta}\right]$ is $\theta$-gauge invariant, i.e., satisfies

$$
i\left(\frac{\partial}{\partial \theta} \frac{\delta}{\delta A_{\theta}^{I}}+f_{I J}^{K} A_{\theta}^{J} \frac{\delta}{\delta A_{\theta}^{K}}\right) \psi^{\text {inv }}\left[A_{\theta}\right]=0
$$

By $\theta$-gauge transformations we mean

$$
\delta A_{\theta}^{I}=D_{\theta} \varepsilon^{I} \quad \delta A_{z}^{I}=f_{J K}^{I} A_{z}^{I} \varepsilon^{K} .
$$

The Hilbert space needs a well-defined scalar product, compatible with Gauss constraint (so, gauge invariant). We then write $\Psi\left[A_{\theta}\right]=\Psi_{\circ}\left[A_{\theta}\right] \psi\left[A_{\theta}\right]$ and look for $\psi\left[A_{\theta}\right]$. This will be done in the spirit of LQG, by changing the focus from the connection $A_{\theta}$ to its holonomy $h\left(\gamma_{z, \theta_{1}, \theta_{2}}\right)=$ $\mathscr{P} \exp \int_{\theta_{1}}^{\theta_{2}} d \theta A_{\theta}$, restricted to paths with constant $z$, otherwise $A_{z}$ would be involved, which is not compatible with our choice of polarization for the wave functional.

The cylindrical space Cyl consists of functionals of the aforementioned form, with $\psi$ being

$$
\psi\left[A_{\theta}\right]=\psi_{\Gamma, f}\left[A_{\theta}\right]=f\left(h\left(\gamma_{z_{1}, \theta_{1}, \theta_{1}^{\prime}}\right), \cdots, h\left(\gamma_{z_{k}, \theta_{k}, \theta_{k}^{\prime}}\right), \ldots, h\left(\gamma_{z_{N}, \theta_{N}, \theta_{N}^{\prime}}\right)\right)
$$

where $\Gamma=\left\{\gamma_{z_{k}, \theta_{k}, \theta_{k^{\prime}}}, k=1 \ldots N\right\}$ is a graph: a finite set of paths. These cylindrical functions, and therefore, our wave functional (apart from that phase factor) depend only on the values that its argument takes on the graph. Finally, the wave functional is a function of a finite number of holonomies (elements of the gauge group $G$ ), which leads us to define the scalar product in Cyl using the invariant Haar measure ${ }^{3} d h_{k}$ :

$$
\left\langle\Gamma, f \mid \Gamma, f^{\prime}\right\rangle=\int \prod_{k=1}^{N} d h_{k} \overline{f\left(h_{1}, \ldots, h_{N}\right)} f^{\prime}\left(h_{1}, \ldots, h_{N}\right)
$$

\footnotetext{
${ }^{3}$ More details and generalization (straightforward) to vectors associated to different graphs are found in [3]
} 
The basis of the kinematical Hilbert space is found with the use of the Peter-Weyl theorem. To every path $\gamma_{z_{k}, \theta_{k}, \theta_{k^{\prime}}}$ on the graph $\Gamma$ we associate a spin $j_{k}$ representation of $G$, and vectors associated to different graphs are orthogonal.

$$
\Psi_{\Gamma, f}\left[A_{\theta}\right]=\Psi_{\circ}\left[A_{\theta}\right] \sum_{\vec{j}, \vec{\alpha}, \vec{\beta}} c_{\beta_{1} \ldots \beta_{N}, j_{1}, \ldots, j_{N}}^{\alpha_{1} \ldots \alpha_{N}} R_{\alpha_{1}}^{j_{1} \beta_{1}}\left(h\left(\gamma_{z_{1}, \theta_{1}, \theta_{1}^{\prime}}\right)\right) \cdots R_{\alpha_{N}}^{j_{N} \beta_{N}}\left(h\left(\gamma_{z_{N}, \theta_{N}, \theta_{N}^{\prime}}\right)\right)
$$

The kinematical Hilbert space (non-separable) is then the direct sum $\mathscr{H}_{\text {kin }}=\bigoplus_{\Gamma} \mathscr{H}_{\Gamma}$, where $\mathscr{H}_{\Gamma}$ is the separable Hilbert space associated with the graph $\Gamma$.

It's possible to define the local operator associated to the holonomy: $\left\langle A_{\theta}\left|\hat{h}\left[A_{\theta}, \gamma\right]\right| \Gamma, f\right\rangle=$ $h\left[A_{\theta}, \gamma\right]\left\langle A_{\theta} \mid \Gamma, f\right\rangle$. The RHS is an element of Cyl associated to a new graph $\Gamma \cup\{\gamma\}$ and a new function $\hat{h}\left[A_{\theta}, \gamma\right] f$.

We impose the constraint in the form of invariance under all finite gauge transformations. This implies that the reduced functional must be functions of the trace of the holonomies along closed paths (cycles, or Wilson loops) $h_{z} \equiv \operatorname{Tr}\left(h\left(\gamma_{z}, \theta, \theta\right)\right)$, which depends on the $z$ coordinate, but not on $\theta$. Thus, each cycle is characterized by its 'height', and the graphs are now sets $C$ of cycles. This defines the Hilbert space $\mathscr{H}_{\text {Gauss }}$, whose basis is the orthonormal set of 'spin network' vectors $|C, J\rangle$, given by

$$
\left\langle A_{\theta} \mid C, J\right\rangle=\Psi_{\circ}\left[A_{\circ}\right] \prod_{k=1}^{N} \chi^{j_{k}}\left(h_{z_{k}}\right) \quad \text { with } \quad \chi^{j}\left(h_{z}\right)=\operatorname{Tr} R^{j}\left(h_{z}\right)
$$

where $J$ stands for $\left(j_{1} \ldots j_{N}\right)$. These vectors are orthonormal, in the sense $\left\langle C, J \mid C^{\prime}, J^{\prime}\right\rangle=\delta_{C, C^{\prime}} \delta_{J, J^{\prime}}$. Now we consider $S_{\circ}$, the space of all finite linear combination of spin-networks, and $\mathscr{H}_{\text {Gauss }}$ is the Cauchy completion of it. It is the direct sum over all $C$ of $\mathscr{H}_{\text {Gauss }}^{C}$, the Hilbert space associate to a graph $C$, which is separable. This is not the case for $\mathscr{H}_{\text {Gauss }}$, since te graphs are indexed by $n$-arrays of real numbers.

Since $|C, J\rangle$ depends on the $z$ coordinate, $\mathscr{H}_{\text {Gauss }}$ is not diffeomorphism invariant. The gauge transformation concerning $z$ diffeomorphisms was not contemplated when we solved Gauss constraint, which must be corrected now. This is done with the use of the group averaging method, based on the Gel'fand triple $S_{\circ} \subset \mathscr{H}_{\text {Gauss }} \subset S_{\circ}^{\prime}$, being $S_{\circ}^{\prime}$ the dual of the spin-networks space. The $z$-diffeomorphism invariant states are shown to be elements of this dual space constructed from any spin-network state through the application of a functional 'projector' $P_{\text {Diff }}: S_{\circ} \rightarrow S_{\circ}^{\prime}$ defined by $\left\langle P_{\text {Diff }} \Psi, \Psi^{\prime}\right\rangle=\sum_{\Psi^{\prime \prime}}\left\langle\Psi^{\prime \prime} \mid \Psi^{\prime}\right\rangle, \quad \forall\left|\Psi^{\prime}\right\rangle \in S_{\circ}$, and the sum is done over all vectors $\left|\Psi^{\prime \prime}\right\rangle$ obtained from $|\Psi\rangle$ by a $z$-diffeomorphism. We conclude that the functionals $P_{\text {Diff }} \Psi$ span the physical Hilbert space $\mathscr{H}_{\text {phys }}$.

The vectors of $\mathscr{H}_{\text {phys }}$ only depend on the equivalence classes of spin-network states under $z$-diffeomorphisms. In particular, a state defined as explained above from $|C, J\rangle$ does not depend on the particular positions $z_{k}$ of the cycles, but only on the number of such cycles and on the spin value associated with each of them. We have then a s-knot state $|J\rangle \equiv\left|j_{1}, \ldots, j_{N}\right\rangle=P_{\text {Diff }}|C, J\rangle$. It worth to mention that one must identify a graph with another, whose cycles (and therefore, spins) are in the reversed order. This is in fact the reason why the second term appears in the following formula for the scalar product of two s-knots:

$$
\left\langle J \mid J^{\prime}\right\rangle=\delta_{N N^{\prime}}\left(\delta_{j_{1} j_{1}^{\prime}} \ldots \delta_{j_{N} j_{N^{\prime}}^{\prime}}+\delta_{j_{N} j_{1}^{\prime}} \ldots \delta_{j_{1} j_{N^{\prime}}^{\prime}}\right)
$$


The s-knots form an orthonormal basis for the physical Hilbert space; they are invariant under all diffeomorphisms, and consist of a solution of the Gauss constraint. Once the set of s-knots is countable, the physical Hilbert space is separable.

\section{Including boundaries}

In order to include boundary contributions to this formalism we consider the $U(1)$ ChernSimons theory on a three-dimensional manifold with the topology $\mathscr{M}=D \times \mathbb{R}$, with $D$ being the disk with boundary $\partial D$, with coordinates $0 \leqslant r \leqslant R, 0 \leqslant \varphi \leqslant 2 \pi$. The action is given by

$$
\mathscr{S}=\frac{\kappa}{4 \pi} \int_{\mathscr{M}}(A \wedge d A)
$$

and the canonical quantization of this model was considered in $[5,6]$. Following their construction ${ }^{4}$ the Gauss law constraint is smeared with the test function $\Lambda^{0}$

$$
G\left(\Lambda^{0}\right)=\frac{k}{2 \pi} \int_{D} \Lambda^{0} \wedge d A
$$

and generates gauge transformations if we consider the boundary condition $\left.\Lambda^{0}\right|_{\partial D}=0$. The observables are defined by

$$
Q(\Lambda)=\frac{k}{2 \pi} \int_{D} d \Lambda \wedge A
$$

being perfectly differentiable in $A$ even if the function $\Lambda$ is nonzero on $\partial D$. Recall that, any gauge invariant object is an observable and hence its Poisson bracket with the Gauss law vanishes. The PB's of $Q(\Lambda)^{\prime}$ s are easy to compute, and read $\left\{Q(\Lambda), Q\left(\Lambda^{\prime}\right)\right\}=\frac{\kappa}{2 \pi} \int_{\partial D} \Lambda \wedge d \Lambda^{\prime}$ showing that they generate a $U(1)$ Kac-Moody algebra on $\partial D$ [7]. It is important to notice that test functions with the same boundary values, up to a constraint, define the same observable,

$$
Q(\Lambda)-Q\left(\Lambda^{\prime}\right)=-G\left(\Lambda-\Lambda^{\prime}\right)
$$

The strategy for quantization is the following. We choose a function

$$
\left.\Lambda_{N}\right|_{\partial D}(\varphi)=\lambda(r) e^{i N \varphi}, N \in \mathbb{Z}, \lambda(0)=0, \lambda(R)=1,
$$

and the polarization in which $A_{\varphi}$ is multiplicative and $A_{r}$ derivative. As in the previous case, where boundary terms were not considered, we observe that there exists a function $\psi_{0}\left[A_{\varphi}\right]=e^{2 \pi i \alpha}$ satisfying $\hat{G}_{\Lambda^{0}} \psi_{0}\left[A_{\varphi}\right]=0$, where $\alpha=\frac{k}{4 \pi} \int_{D} d^{2} x\left(g^{-1} \partial_{r} g g^{-1} \partial_{\varphi} g\right)$ and

$$
\delta \alpha=\frac{k}{4 \pi} \int_{D} d^{2} x\left\{\partial_{r}\left(g^{-1} \delta g w_{\varphi}\right)-2 g^{-1} \delta g \partial_{r} w_{\varphi}\right\}
$$

Calling $Q_{N}$ the quantum operator for $Q\left(\Lambda_{N}\right)$ and applying on the function $\psi_{0}\left[A_{\varphi}\right]$ we get

$$
\begin{aligned}
Q_{N} \psi_{0}\left[A_{\varphi}\right] & =\frac{k}{4 \pi} \Lambda_{N}\left(R, \varphi_{0}\right) \int_{\partial D} d \varphi A_{\varphi} \psi_{0}\left[A_{\varphi}\right]+\frac{k}{4 \pi} \int_{\partial D} d \varphi \Lambda_{N}(R, \varphi) A_{\varphi} \psi_{0}\left[A_{\varphi}\right] \\
Q_{M} Q_{N} \psi_{0}\left[A_{\varphi}\right] & =\frac{k}{4 \pi} \Lambda_{N}\left(R, \varphi_{0}\right) \int_{\partial D} d \varphi A_{\varphi} Q_{M} \psi_{0}\left[A_{\varphi}\right]+\frac{k}{4 \pi} \int_{\partial D} d \varphi \Lambda_{N} A_{\varphi} Q_{M} \psi_{0}\left[A_{\varphi}\right] \\
& +\frac{i k}{8 \pi} \int_{\partial D} d \varphi \Lambda_{N}(R, \varphi) \partial_{\varphi} \Lambda_{M} \psi_{0}\left[A_{\varphi}\right]
\end{aligned}
$$

\footnotetext{
${ }^{4}$ For other approaches to this problem see [8] and references therein.
} 
and we have that [7]

$$
\left[Q_{N}, Q_{M}\right]=N k \delta_{N+M, 0}
$$

is verified at quantum level.

\section{Conclusions}

As it was mentioned in the introduction, we found here a great opportunity to apply the LQG scheme, and the most pleasant fact is that due to the simplicity of the theory, one can really see what is going on at each step ${ }^{5}$. It is interesting how LQG's approach fitted well in the proposal given in [2] for the construction of a wave functional. So, what we have done here is basically a continuation of [2], not only finding such functional, but also defining the physical Hilbert space and a compatible scalar product.

Concerning the boundary inclusion, our next step is the construction of the Hilbert space spanned by the aforementioned states. Once this aim is achieved, we will expand our analysis to non-abelian groups in order to apply the method not only for non-abelian Chern-Simons theory but for $3 \mathrm{~d}$ gravity as well. Other types of topology may be also studied [9].

\section{References}

[1] C. Rovelli, Quantum gravity, Cambridge University Press, Cambridge, 2004 [arXiv:gr-qc/9710008 ]

[2] G.V. Dunne,R. Jackiw, C.A. Trugenberger, Chern-Simons theory in the Schrödinger representation, Annals of Physics, v. 194, p. 197-223, n. 1, 1989

[3] C. P. Constantinidis, G. Luchini, O. Piguet, The Hilbert space of Chern-Simons theory on the cylinder. A loop quantum gravity approach, Class.Quant.Grav. v. 27, n. 6, 2010 [arXiv: gr-qc/0907.3240]

[4] G.V. Dunne, Aspects of Chern-Simons in proceedings of Les Houches Summer School In Theoretical Physics, Session 69: Topological aspects of low dimensional systems, [arXiv: hep-th/9902115]

[5] A. P. Balachandran et al., Conformal edge currents in Chern-Simons theories, Int.J.Mod.Phys.A7:4655-4670,1992 [arXiv:hep-th/9110072].

[6] A. P. Balachandran, Gauge symmetries, topology and quantization, in proceedings of Miniworkshop on Methods of Electronic Structure Calculations, AIP Conf.Proc.317:1-81,1994 [arXiv:hep-th/9210111].

[7] For a review, see P. Goddard, D. Olive, Kac-Moody and Virasoro algebras in relation to quantum physics, Int. J. Mod. Phys. A1, 303 (1986).

[8] S. Carlip, Conformal field theory, (2+1)-dimensional gravity, and the BTZ black hole, Class.Quant.Grav.22:R85-R124,2005 [arXiv:gr-qc/0503022]

[9] C.P. Constantinidis, O.Piguet, A.Rios, work in progress.

\footnotetext{
${ }^{5}$ In [3] the reader can find some pictures that clarify the spin-networks construction.
} 